\title{
O TRABALHO COTIDIANO DA ENFERMEIRA NA SAÚDE DA FAMÍLIA: UTILIZAÇÃO DE FERRAMENTAS DA GESTÃO ${ }^{1}$
}

\author{
Lauren Suemi Kawata², Silvana Martins Mishima³, Mara Quaglio Chirelli4, Maria José Bistafa Pereira ${ }^{5}$
}

\footnotetext{
${ }^{1}$ Pesquisa financiada pelo CNPq.

${ }^{2}$ Doutoranda do Programa de Pós-Graduação em Enfermagem em Saúde Pública da Escola de Enfermagem de Ribeirão Preto (EERP) da Universidade de São Paulo (USP). Professora do Curso de Graduação em Enfermagem da Universidade Federal de Uberlândia. Minas Gerais, Brasil. E-mail: lsuemi@hotmail.com

${ }^{3}$ Livre-docente. Professor Associado da EERP/USP. São Paulo, Brasil. E-mail: smishima@eerp.usp.br

${ }^{4}$ Doutor em Enfermagem. Professor Doutor da Faculdade de Medicina de Marília. São Paulo, Brasil. E-mail: marachirelli@ gmail.com

${ }^{5}$ Livre-docente. Professor Doutor da EERP/USP. São Paulo, Brasil. E-mail: zezebis@eerp.usp.br
}

\begin{abstract}
RESUMO: Estudo descritivo, qualitativo, que objetivou identificar e analisar os atributos mobilizados pela enfermeira no trabalho cotidiano na Saúde da Família voltados ao planejamento, a coordenação e a supervisão, visando uma aproximação aos desempenhos na construção da competência gerencial. O cenário constituiu-se em quatro Unidades de Saúde da Família de Ribeirão Preto-SP. Os sujeitos foram quatro enfermeiras com mais de um ano de trabalho. A coleta de dados foi realizada de junho a julho de 2006, através da observação participante, totalizando 160 horas. Optamos pela análise de conteúdo, utilizando a técnica de análise temática. Observamos que a supervisão realizada é caracterizada pelos aspectos de controle e educação; o planejamento apresenta-se como impulsionador de práticas voltado para articulação dos processos de trabalho juntamente com o exercício da coordenação. Concluímos que as enfermeiras mobilizam o saber aprender e o saber conviver.
\end{abstract}

DESCRITORES: Enfermagem. Competência profissional. Saúde da família. Organização e administração.

\section{NURSING DAILY WORK IN FAMILY HEALTH: USING MANAGEMENT TOOLS}

\begin{abstract}
The purpose of this qualitative, descriptive study was to identify and analyze the attributes mobilized by nurses when developing their day-to-day Family Health work regarding planning, coordination, and supervision, seeking an approximation with performances in constructing managerial competence. The setting consisted of four Family Health Units in the city of Ribeirão Preto, SP, Brazil. The subjects were four nurses who had worked for at least one year with the Family Health Program. Data was collected from June to July of 2006 through participant 160 hours of observation. Data analysis was performed through content analysis, using the thematic analysis technique. It was observed that supervision is characterized by aspects of control and education and that planning boosts practices aimed at articulating work processes while exercising coordination. We conclude that nurses mobilize knowing how to learn and knowing how to live together.
\end{abstract}

DESCRIPTORS: Nursing. Professional competence. Family health. Organization and administration.

\section{EL TRABAJO DIARIO DE LA ENFERMERA EN LA SALUD FAMILIAR: LA UTILIZACIÓN DE INSTRUMENTOS DE GESTIÓN}

RESUMEN: Estudio descriptivo, cualitativo, cuyos objetivos fueron identificar y analizar los atributos utilizados por la enfermera al realizar su trabajo diario en la Salud Familiar, los cuales se basaron en la planificación, coordinación y supervisión, con la finalidad de aproximar el desempeño y así construir la competencia gerencial. El estudio fue realizado en cuatro Unidades de Salud Familiar en Ribeirão Preto, SP, Brasil. Los sujetos fueron: cuatro enfermeras con más de un año de trabajo en Salud Familiar. La recolección de los datos fue hecha de junio a julio de 2006 por medio de observación participativa, totalizando 160 horas. Se empleó el análisis de contenido, utilizando la técnica de análisis temático. Observamos que la supervisión se caracteriza por aspectos como control y educación, la planificación incentiva las prácticas basadas en la articulación de los procesos de trabajo, los cuales son realizados conjuntamente con la coordinación. Concluimos que las enfermeras utilizan el saber aprender y el saber convivir.

DESCRIPTORES: Enfermería. Competencia profesional. Salud de la familia. Organización y administración. 


\section{INTRODUÇÃO}

O trabalho de enfermagem, compreendido como prática social configura-se como ação produtiva marcado por determinantes históricos, políticos, sociais e econômicos.

No contexto brasileiro, a partir da década de 90, de busca pela implementação do Sistema Único de Saúde (SUS), a Saúde da Família (SF) constituise em uma estratégia de política pública que tem se conformado e consolidado como um campo de atuação para a enfermeira, já que em dezembro de 2008, havia 29.057 equipes de SF implantadas no país, o que correspondia a uma cobertura de $49,51 \%$ da população brasileira. ${ }^{1}$

Com a SF, espera-se que haja substituição do modelo assistencial de cunho curativo, centrado no hospital para um modelo sustentado pelos princípios da Atenção Primária à Saúde (APS), cujo foco é a vigilância em saúde da população adscrita a um território, com a implementação de uma lógica de produção de cuidados em que as ações de saúde desenvolvidas sejam permeadas por vínculo, acolhimento e responsabilização pela equipe de saúde. ${ }^{2}$

O documento inicial do Ministério da Saúde (MS), ao trazer a questão da equipe multiprofissional como central para o desenvolvimento da $\mathrm{SF}$, apresenta um rol de atribuições para cada um dos trabalhadores de saúde da equipe, necessário certamente para a delimitação das funções, mas não suficiente para possibilitar a articulação do trabalho na direção dos princípios colocados para a proposta da SF. ${ }^{3} \mathrm{O}$ MS aponta como atribuição do enfermeiro "planejar, gerenciar, coordenar, executar e avaliar a USF", $4: 76$ o que traz à discussão os aspectos gerenciais da equipe e da unidade, que não pode ficar restrita às atividades administrativas burocráticas, estabelecendo como função básica apenas o controle do trabalho, mas considerar as dimensões que a atividade gerencial apresenta, principalmente quando se tem como norte a estratégia como disparadora da mudança de modelo assistencial.

Mais recentemente, a Política Nacional de Atenção Básica estabelece que à equipe de SF cabe o desenvolvimento de um processo de trabalho voltado para o planejamento, organização e execução de ações de saúde no território, sendo que a enfermeira, enquanto membro integrante da equipe multidisciplinar na SF, deve apresentar competências que possibilitem o estabelecimento de ações comprometidas com a reversão do mode- lo assistencial vigente e, conseqüentemente, com a consolidação do SUS. ${ }^{5}$

É importante considerarmos que embora desejável, a noção de equipe colocada pelo MS apresenta uma perspectiva de recomposição do trabalho coletivo no nível discursivo, pois no diaa-dia dos serviços não basta a definição de um perfil de atividades a serem desenvolvidas para se garantir tal recomposição do trabalho individual e autônomo dos trabalhadores na direção da construção prática do trabalho em equipe. ${ }^{6}$

A gerência, neste sentido, pode ser um instrumento com potência para desencadear no conjunto dos trabalhadores um processo de reflexão e revisão de sua prática, que encaminhe para a adesão e comprometimento a um processo de produção de cuidados à saúde e, não de cumprimento de tarefas fragmentadas centradas no desenvolvimento de procedimentos isolados. Parece que este (re)pensar do trabalho em saúde, aí tomando o trabalho na SF, se impõe na direção de efetivar mudanças significativas no sistema de saúde caso haja uma participação de todos os profissionais de saúde para a (re)organização dos serviços. ${ }^{7-8}$

Assim, considerando a gestão como uma ferramenta cuja dimensão estratégica pode possibilitar a transformação do processo de trabalho em saúde e em enfermagem, ${ }^{6}$ este estudo focará o planejamento, a supervisão e a coordenação enquanto instrumentos relativos à área de competência gerencial da enfermeira, a partir da compreensão da gestão e gerência enquanto conceitos sinônimos.

É importante ressaltarmos que a noção de competência apresenta um caráter polimorfo e polissêmico, sendo que, na presente investigação, estamos considerando os conceitos teóricos de competência dialógica que articula o mundo do trabalho e formação e que " [...] trabalha com o desenvolvimento de capacidades ou atributos (cognitivos, psicomotores e afetivos)" 9:372 Ainda, destacamos que a competência não é observada diretamente, mas sim é inferida pelo desempenho que consiste na combinação de atributos (conhecimentos, habilidades e atitudes) que fundamentam a realização de atividades profissionais. ${ }^{10}$

A definição das competências necessárias à enfermeira e o estabelecimento de mecanismo para o desenvolvimento das mesmas tem se constituído enquanto um desafio, ${ }^{11}$ sendo que, ao pensarmos o trabalho da enfermeira na SF, isto não se coloca de forma diferente.

Adicionalmente, a necessidade de parcerias com os serviços para o desenvolvimento de pes- 
quisas e para aplicação do conhecimento tem sido enfatizada, destacando a relação entre formação e serviços, com a finalidade de criação de estratégias de mudanças dentre as quais está inserido o ensino baseado em competência.

Ainda, investigações que focam supervisão, coordenação e planejamento como ferramentas utilizadas pelas enfermeiras têm sido desenvolvidas predominantemente voltadas ao ambiente hospitalar. Desse modo, o presente estudo tem por objetivos: identificar e analisar os atributos mobilizados pela enfermeira no desenvolvimento do trabalho cotidiano na SF voltados ao planejamento, a coordenação e a supervisão, visando uma aproximação aos desempenhos na construção da competência gerencial.

\section{MÉTODO}

Este estudo apresenta um caráter descritivo, na abordagem qualitativa. O campo de estudo consistiu no município de Ribeirão Preto-SP, contando com uma população de 563.912 habitantes, sendo considerado referência nacional para o setor saúde, devido à presença de grandes centros de ensino e pesquisa. ${ }^{12} \mathrm{~A}$ rede básica de saúde do município é composta por 27 Unidades Básicas de Saúde (UBSs)* , cinco Unidades Básicas e Distritais (UBDs) $^{* *}$, um Ambulatório Regional de Especialidades, um Ambulatório de Especialidades Pediátricas, um Ambulatório Regional de Saúde Mental, dois Núcleos de Assistência Psico-social e 22 equipes de SF.

O cenário da pesquisa se constituiu em quatro das cinco Unidades de Saúde da Família (USFs) ligadas à Universidade de São Paulo (USP), sendo o critério de seleção utilizado, a "antigüidade" da unidade na implantação da SF no município, isto é, aquelas USFs há mais tempo instaladas e em funcionamento. Destacamos que uma das USFs não foi selecionada para a pesquisa por se tratar de uma unidade mista, ou seja, uma UBS, com suas atividades próprias e adicionalmente, uma equipe de SF. Ainda, ressaltamos que em cada USF há uma equipe de SF composta por: um médico generalista, uma enfermeira, duas auxiliares de en- fermagem, um dentista, cinco agentes comunitários de saúde e um auxiliar de serviços gerais.

Os sujeitos da pesquisa foram as enfermeiras com mais de um ano de trabalho na SF, num total de quatro, fato que indicaria certo acúmulo de experiências no trabalho pelas mesmas.

A coleta de dados foi realizada no período de junho a julho de 2006, através da observação participante do trabalho das enfermeiras, em uma semana típica de trabalho (40 horas), isto é, semana na qual não ocorreu qualquer motivo que interferisse na rotina de trabalho das enfermeiras, tais como feriados, campanhas de saúde de qualquer natureza, etc, perfazendo um total de 160 horas de observação. Foram observadas: as atividades desenvolvidas e como as mesmas eram realizadas; a tomada de decisão (com quais critérios e recursos); a interação (com os usuários e a equipe); o tipo e o modo da comunicação adotada, entre outros. As observações foram registradas em um diário de campo, considerando tanto as atividades realizadas quanto os aspectos físicos e sociais, ou seja, aspectos relacionados à estrutura física, presença de barreiras (como barulho) e interação. Portanto, os registros apresentaram a descrição das tarefas realizadas pelas enfermeiras e todas as manifestações (verbais, não verbais, atitudes) observadas que foram mobilizadas, nos aproximando de uma apreensão de todo o processo.

Para a análise dos dados, as observações foram organizadas de modo a se constituir o corpus da pesquisa, sendo que optamos pela análise de conteúdo, utilizando a técnica da análise temática. ${ }^{13-14}$ Inicialmente, foram realizadas várias leituras no tratamento do material registrado das observações, buscando-se uma maior aproximação com o mesmo que, posteriormente, foi separado em fragmentos sistematizados nos seguintes aspectos: o que faz (para identificar qual a ação desenvolvida), como faz (a fim de buscar uma aproximação aos atributos) e para que faz (visando indicar a área de competência).

Ressaltamos que a noção de competência apresenta várias abordagens, dentre elas: a condutivista, a funcionalista e a construtivista. ${ }^{9}$ Desse

* UBSs são unidades localizadas em territórios específicos, contando com atendimento médico nas especialidades básicas (pediatria, clínica médica, ginecologia e obstetrícia), atendimento odontológico e de enfermagem, realização de procedimentos e imunização para uma população de cerca de 10.000 a 15.000 habitantes.

** UBDs são unidades de referência para uma região distrital, realizando atendimentos especializados (p.ex. neurologia, ortopedia, endocrinologia, cardiologia) referenciados pelas UBS de sua região distrital. Conta ainda, com serviço de urgência 24 horas e serviços de apoio diagnóstico. 
modo, reiteramos que, nesta pesquisa, seguimos o referencial teórico de competência dialógica que considera o contexto e a cultura local onde se realiza a ação. Assim, a identificação do desempenho (mobilização de uma rede de atributos, combinando-os de diferentes formas para resolver as situações cotidianas de trabalho) da enfermeira no desenvolvimento do trabalho cotidiano na SF na área de competência da gestão do trabalho, possibilita captar os seus atributos, ou seja, o uso que a enfermeira faz daquilo que sabe, sente, vive e observa para realizar gestão, incorporando também a ética e os valores.

Para o desenvolvimento da pesquisa, foram seguidas as normatizações da Comissão Nacional de Ética em Pesquisa, de acordo com a Resolução No 196/96 do Conselho Nacional de Saúde. O projeto apresentou a aprovação do Comitê de Ética em Pesquisa do Centro de Saúde Escola da Faculdade de Medicina de Ribeirão Preto sob o ${ }^{\circ}$ 0192. Os sujeitos concordaram e deram anuência no Termo de Consentimento Livre e Informado. No tratamento do material empírico, buscamos preservar a identidade das enfermeiras, sujeitos da pesquisa, identificando-as por cores.

\section{RESULTADOS E DISCUSSÃO}

A supervisão, compreendida como parte do processo coletivo do trabalho em saúde, relacionase às demandas e aos objetivos dos serviços e, portanto, consiste em um produto tanto de políticas institucionais e estruturais organizativas quanto de uma prática em que se reproduz e se constrói essas políticas, e apresenta três aspectos: político, de controle e de educação. ${ }^{15}$ Nesse sentido, na presente investigação, a supervisão realizada pelas enfermeiras é caracterizada principalmente pelos aspectos de controle e educação.

A dimensão do controle aparece no trabalho das enfermeiras recorrentemente. A finalidade que assume é no sentido estrito de controlar os trabalhadores, (Agente Comunitário de Saúde-ACS, médicos residentes, auxiliares de enfermagem), predominantemente quanto ao cumprimento de horário e jornada de trabalho, o que está relacionado à origem da supervisão no processo de trabalho, decorrente da divisão técnica e social que gerou a necessidade de existir uma atividade que assegurasse que normas e regras fossem cumpridas.

Os atributos mobilizados pelas enfermeiras para exercer tal controle envolvem: atitudes que podem indicar valorização do outro e preocupa- ção com direitos e deveres no e para o trabalho; conhecimentos acerca: da saúde do trabalhador, da dinâmica e da rotina da unidade, de princípios éticos (como justiça, tolerância e responsabilidade), das atribuições de cada trabalhador; habilidades relativas ao tipo de linguagem, à tomada de decisão e à interação.

As observações indicaram pistas que, em determinados momentos ao realizar o controle, as enfermeiras fazem uma avaliação do trabalho desenvolvido pelos trabalhadores e possíveis alterações no processo de trabalho da equipe, considerando a totalidade do serviço, a fim de que a atenção ao usuário não seja prejudicada. Ao mesmo tempo, não há evidências que as enfermeiras levam os trabalhadores a refletirem quanto ao controle. Assim, não está claro se existe articulação da dimensão do controle à organização do trabalho para todos os trabalhadores.

[...] Eu preciso contar as horas, mas antes preciso conversar com a ACS. Auxiliar de Enfermagem, chama a ACS, por favor! Fala pra ela vir aqui. [ACS entra na sala e enfermeira Branca pede pra ela fechar a porta]. ACS, tô vendo essa história de horas .... Lembrei que não fiz as contas porque você tem chegado bem mais cedo. Se você chegasse e trabalhasse, mas não é isso que acontece [o ACS responde que anota o horário que chega, porque tem que anotar, mas que não é para contar a hora]. Tinha um tempo que você chegava mais cedo porque a agente de serviços gerais tava de férias. Aí conta hora. Mas agora com [...], não vai ficar fazendo isso (Enfermeira Branca).

A educação, outro componente do processo de supervisão, ${ }^{15}$ está presente no trabalho das enfermeiras em relação à capacitação das próprias enfermeiras e ao treinamento da equipe. Identificamos que, no cotidiano de seu trabalho, nem sempre há indícios que a educação desenvolvida apresenta o caráter de educação permanente, isto é, constituindo-se em um recurso estratégico para gestão do trabalho e recomposição das práticas de formação e atenção. ${ }^{16}$

Observamos que as enfermeiras, ao promover e buscar educação mobilizam atributos relacionados a conhecimentos clínicos, técnicos e organizacionais; habilidades para articulação com outros níveis de atenção e atitudes que envolvem princípios de responsabilidade e preocupação ética.

[Durante reunião de equipe] Põe na pauta da semana que vem, Ética. Eu vou buscar um texto. Uma vez nós fizemos discussão no Cuiabá [UBDS de referência da USF], falando de ética profissional e ética das relações [Médica fala sobre ética]. Eu acho 
que você tá certa! Às vezes, o meu papel, o seu papel é chato. Eu não posso ser agradável e omissa. Acho que ninguém aqui fica à toa. Mas, em alguns momentos, a gente esquece. A gente quando visita outro serviço, a gente olha. [...] Não dá pra representante ficar na recepção e a gente conversando com paciente. O caso da agente de serviços gerais, a gente não pode excluir. Eu já vi vários casos na frente dela. Atendeu telefone no meio da orientação, eu já fiz isso. Mas precisa prestar atenção! Algumas coisas a gente precisa falar: as serventes da Cuiabá reclamaram, disseram que não vão fazer limpeza de sujeira crônica. A paciente chegou pra mim e falou: nossa, não dá pra clarear esse banco? Tá encardido! Enfim, é coisa pra todos nós. Não estou me excluindo. Às vezes, a gente entra na recepção tomando café. Outro dia o computador tava manchado de café. É o unico que temos (Enfermeira Verde).

Assim, a supervisão pode ser um instrumento utilizado para promover o envolvimento dos trabalhadores com os objetivos do projeto do qual fazem parte, buscando responsabilização, cumprimento às questões éticas presentes no trabalho e educação permanente. Compreendemos que a forma como as enfermeiras combinam seus atributos para realizarem a supervisão pode ser fundamental para a implementação efetiva da SF.

Outro instrumento do processo de trabalho gerencial da enfermeira na SF é o planejamento que consiste em um conjunto de conhecimentos teóricos, práticos e organizacionais, que possibilita a programação de estratégias e ações necessárias a partir de uma dada realidade, para alcançar os objetivos propostos. Desse modo, o planejamento tem ganho importância como instrumento/ atividade dos processos de gestão (considerando a necessidade de articulação dos processos de trabalho), impulsionador de práticas sociais transformadoras (buscando novas relações sociais) e método de ação governamental (referido à produção de políticas). ${ }^{17}$

Nesse estudo, o planejamento, enquanto ferramenta do trabalho da enfermeira apresentase como impulsionador de práticas e mais voltado para a articulação dos processos de trabalho.

Como as USFs nas quais as enfermeiras atuam são vinculadas à USP e, portanto, constituemse como serviço-escola, identificamos vários momentos em que há evidente preocupação destas em considerar a inserção de estudantes na unidade e em desenvolver um planejamento das atividades a serem realizadas pelos mesmos:

[Durante reunião de equipe] Médico, hoje chegam os alunos, né? Tem alguma coisa? [Médico fala sobre a distribuição dos alunos para Visita Domiciliar (VD)]. Você tem a escala de quem vai te cobrir? Porque cada dia vai ser um. São 3 pessoas que vão te cobrir. Até porque se algum faltar, a gente sabe pra quem ligar. [Enfermeira Azul anota a escala]. Sabe por que eu tô te perguntando? O perfil do docente da FMRP é de atendimento médico. E você tá programando pros alunos outro tipo de atividade: VD. Então, ele pode mudar né! [Médico responde] Médico, mas aí esses contratados vão ficar com a gente no $E V$ [consulta médica eventual]? Porque você desmarcou os pacientes, mas a unidade vai tá aberta e o pessoal vai procurar. Eles reconhecem que, além de cobrir você por conta dos alunos, tem um serviço por trás, uma população? Ficou assim [mostra a escala no papel] Vou pôr lá no mural (Enfermeira Azul).

Para o planejamento de atividades, as enfermeiras, em certos momentos, identificam a necessidade da elaboração do plano, coordenam, articulam e descrevem possibilidades, identificam os atores e os recursos envolvidos com o plano, tentando viabilizá-lo, realizando previsão de situações e possíveis alternativas para a tomada de decisão.

Um elemento importante identificado, refere-se ao fato das enfermeiras considerarem o sistema de informação um instrumento essencial para o conhecimento da realidade demográfica e epidemiológica, o qual viabiliza o gerenciamento, já que possibilita subsidiar o planejamento e a tomada de decisão e promover mudanças nas intervenções no nível individual e coletivo.

Observamos que as enfermeiras também programam atividades de educação permanente. Ao fazer isso, mobilizam atributos, provavelmente considerando a importância e finalidade da ação para o trabalho da equipe.

Além de planejar atividades de ensino a estudantes e de educação permanente, identificamos que predomina no cotidiano do trabalho das enfermeiras o desenvolvimento de projetos para o cuidado individual. Destacamos que, no trabalho das enfermeiras, a programação oscila entre momentos em que é desenvolvida de modo mais participativo e períodos em que realiza pequenas colaborações a fim, possivelmente, de qualificar a prática a ser realizada por outros trabalhadores, já que o planejamento consiste na arte de fazer escolhas e de desenvolver planos que favoreçam o processo de intervenção. ${ }^{17}$ Planos esses que para serem realizados com possibilidades de ter sucesso, necessitam de sistema de direção comunicativo e de definições claras de responsabilidades. ${ }^{18}$ 
Desse modo, as enfermeiras, quando não estão responsáveis pela execução das tarefas, auxiliam os demais trabalhadores provavelmente para que haja sincronia e coerência na execução das ações planejadas. Ainda, ressaltamos a importância do planejamento participativo, pois o desenvolvimento do mesmo consiste em espaços de trocas e reflexão crítica, momentos de compartilhar poder, capacitar trabalhadores, subsidiar construção de subjetividades e estimular a definição de projetos coletivos e solidários. ${ }^{19-20}$

Ainda, identificamos que, em raros momentos, as enfermeiras desenvolvem o planejamento de ações para a comunidade, embora este seja um foco bastante enfatizado na Estratégia de Saúde da Família (ESF). ${ }^{5}$ Um exemplo desta observação foi a programação de atividades de férias para a população tanto na unidade como em articulação como outras instituições, o que indica que as enfermeiras possivelmente manejam valor acerca da intersetorialidade e a integralidade no cotidiano do serviço, princípios norteadores da SF. ${ }^{5}$

[Equipe discutindo sobre o público alvo da atividade] Por outro lado, a gente precisa até checar. Aquele projeto de extensão. A terapia ocupacional ia fazer com capoeira e aí vem um valor [...] No princípio, a gente não sabe como responder. Dá pra pensar no trabalho voluntário. Agora pras férias você poderia pensar em 2 dias da semana? [A médica pergunta se não iria atrapalhar o projeto da Terapia Ocupacional] Podíamos combinar das três às cinco porque abre às duas e se marcar às duas, eles vão chegar mais cedo. [sobre o critério de participação] Da área. Não precisa ser cadastrado porque metade da área não é cadastrada. [...] São duas faixas etárias, não precisa ser separado? [...] Se a gente conseguisse aproveitar essa meia hora pra fazer a lista. Se não tiver o número, a pessoa que for ligar procura no prontuário. Tudo a gente divulga [Equipe falando sobre outras atividades de férias no Núcleo]. Não vamos montar um projeto. Dá pra gente pensar naquilo que a ACS falou. Trazer um telão e passar filme [...] (Enfermeira Rosa).

Ressaltamos que, freqüentemente, as enfermeiras aproveitam o momento de reunião de equipe para realizar também o planejamento de serviços como, por exemplo, o envio de relatórios e coordená-los.

Ao planejar as atividades, as profissionais mobilizam conhecimentos sobre: estrutura física da unidade, condição da população adscrita, regionalização, sistema de informação, localização de documentos na unidade, experiências anteriores; demonstram habilidade para conduzir o plane- jamento, coordenar o fluxo interno da unidade, priorizar grupos de risco, distribuir tarefas e para articular o trabalho com outros trabalhadores da equipe; têm atitudes de realizar sugestões para tomada de decisões e de interação.

Considerando que a coordenação consiste em um instrumento que define claramente as responsabilidades das pessoas envolvidas no processo, visando facilitar o alcance das finalidades propostas, de modo que possibilite redução dos atritos e a condução do processo de trabalho mais colaborativo ${ }^{17}$ identificamos que as enfermeiras desenvolvem o planejamento de ações para a organização do cuidado juntamente com o exercício da coordenação.

No conjunto das atividades identificadas, chama atenção a ausência de um processo mais sistemático de avaliação realizado pelas profissionais, ou mesmo pela equipe, o que também foi observado em outro estudo ${ }^{6}$ em relação à gerência nas USFs. Mesmo considerando que o período de observação se restringiu a uma semana típica de trabalho na unidade de saúde, no conjunto das observações não identificamos claramente a avaliação das ações individuais ou coletivas, ou mesmo voltadas para a organização dos serviços.

É importante destacarmos que os elementos aqui apontados em relação ao trabalho gerencial da enfermeira na SF, estão separados para fins de análise, uma vez que no cotidiano a mobilização dos atributos se dá de forma intimamente articulada.

\section{CONSIDERAÇÕES FINAIS}

Neste estudo, ao buscarmos identificar e analisar os atributos voltados ao planejamento, a coordenação e a supervisão, mobilizados pela enfermeira no desenvolvimento do trabalho, verificamos que estas atividades são freqüentemente desenvolvidas de modo centralizado, indicando que, apesar de a equipe estar presente, a tomada de decisão pode ser realizada sem participação efetiva de outros trabalhadores. Ainda, em certos momentos, a comunicação desenvolvida pelas enfermeiras efetiva-se somente através da transmissão de informação. Estes aspectos apontam para o desenvolvimento da ação gerencial de forma pouco participativa, sem que esteja presente a dimensão comunicativa do trabalho gerencial.

Concluindo, inferimos que as enfermeiras, mobilizam o saber aprender ao buscarem outras formas de saber/conhecer a atenção à saúde na ESF, e o saber conviver, ao lidarem com a 
diversidade de trabalhadores na saúde, mesmo apontando contradições como as assinaladas na forma ainda centralizada de atuarem. Saberes estes que podem contribuir para ampliar o leque de intervenções de enfermagem sobre o processo saúde-doença-cuidado.

Para finalizar, reiteramos que a captação dos atributos mobilizados pelas enfermeiras para o planejamento, a coordenação e a supervisão ocorreu a partir do olhar das pesquisadoras e, portanto, esses atributos podem e devem ser discutidos com as enfermeiras, a fim de compreendermos seus determinantes, considerando o contexto das situações e envolvendo o projeto proposto pelo município e suas possibilidades de viabilização no cotidiano.

Retomamos aqui a necessidade de parcerias com os serviços para o desenvolvimento de pesquisas e para aplicação do conhecimento, destacando a relação entre formação e serviços, com a finalidade de criação de estratégias de mudanças dentre as quais está inserida a formação baseada em competência.

Destacamos que a capacidade de mobilização e convocação dos atributos necessários para atuar face a uma situação, articulando-os de forma pertinente e oportuna, seria a própria essência da competência. ${ }^{21}$ Portanto, não estamos falando somente de aplicação de recursos, mas da mobilização de atributos (conhecimentos, habilidades e atitudes), combinados de diferentes formas, frente a uma dada situação da prática profissional, de acordo com o grau de autonomia e domínio do profissional na ação, ou seja, o desempenho da enfermeira, a parte visível da competência, a ser captado na ação.

No entanto, há diversas lógicas de discussão, e a que nos referimos aponta para uma determinada forma de compreensão de competência, a qual constrói o diálogo entre a formação e o mundo do trabalho. Na competência dialógica buscamos a reflexão sobre a ação. Neste sentido, ao trabalharmos na formação com orientação por competência dialógica, estamos privilegiando a integralidade na construção do conhecimento, a relação da teoria e da prática, a práxis.

O enfoque dialógico da competência numa perspectiva mais integradora considera também o contexto e a cultura local de trabalho, onde se dá a ação, incorporam a ética e os valores como elementos do desempenho. ${ }^{9,22}$

Neste processo de formação, que se dá "no" e "para o" processo de trabalho cotidiano dos serviços, o foco principal é produzir o cuidado em saúde a partir da prática profissional, construindo sentidos para a ação da enfermeira, onde esta possa identificar a finalidade do trabalho e mobilizar os atributos de forma combinada, para a produção da ação em saúde e em enfermagem, de acordo com o projeto político que se tem em marcha.

\section{REFERENCIAS}

1. Ministério da Saúde (BR), Atenção Básica e a Saúde da Família: números [página da internet]. Brasília (DF): MS, 2009 [atualizado 2009 Mar 26; acesso em 2009 Mar 26]. Disponível em: http:/ / dtr2004.saude. gov.br/dab/abnumeros.php\#mapas

2. Starfield B. Atenção Primária. Equilíbrio entre necessidades de saúde, serviços e tecnologia. Brasília (DF): UNESCO/MS; 2002.

3. Ministério da Saúde (BR), Secretaria de Atenção à Saúde, Departamento de Atenção Básica. SF: uma estratégia para a reorientação do modelo assistencial. Brasília (DF): MS; 1998.

4. Ministério da Saúde (BR), Secretaria de Atenção à Saúde, Departamento de Atenção Básica. Guia Prático do Programa de SF. Brasília (DF): MS; 2001.

5. Ministério da Saúde (BR), Secretaria de Atenção à Saúde, Departamento de Atenção Básica. Política Nacional de Atenção Básica. Brasília (DF): MS; 2007.

6. Mishima SM. A gerência de serviços de atenção primária à saúde como instrumento para a reorganização da assistência à saúde - o caso do Programa de SF [tese de livre docência]. Ribeirão Preto (SP): Universidade de São Paulo. Escola de Enfermagem de Ribeirão Preto; 2003.

7. Campos GWS. Reforma da reforma: repensando a saúde. São Paulo (SP): Hucitec; 1992.

8. Campos GWS. Um método para a análise e co-gestão de coletivos. São Paulo (SP): Hucitec; 2000.

9. Lima VV. Competência: distintas abordagens e implicações na formação de profissionais de saúde. Rev Interface. 2005 Mar-Ago; 9(17):369-79.

10. Ribeiro ECO, Lima VV. Competências profissionais e mudanças na formação. Olho Mágico 2003 AbrJun; 10(2):47-52.

11. Cunha ICKO, Ximenes Neto FRG. Competências gerencias das enfermeiras: um novo velho desafio? Texto Contexto Enferm. 2006 Jun-Set; 15(3):479-82.

12. Fundação Sistema Estadual de Análise de Dados [página na internet]. São Paulo (SP); 2008 [atualizado em 2008 Set 05; acesso em 2008 Set 07]. Disponível: em www.seade.gov.br

13. Bardin L. Análise de conteúdo. Lisboa (PT): Edições 70, 1995.

14. Minayo MC. O desafio do conhecimento: pesquisa qualitativa em saúde. $6^{\text {a }}$ ed. São Paulo/Rio de Janeiro (SP/RJ): Hucitec/ABRASCO; 1999. 
15. Silva EM. Supervisão do trabalho de enfermagem em saúde pública no nível local. [tese] Ribeirão Preto (SP): Universidade de São Paulo. Escola de Enfermagem de Ribeirão Preto; 1997.

16. Ministério da Saúde (BR), Secretaria da Gestão do Trabalho e da Educação na Saúde, Departamento de Gestão na Educação na Saúde. Curso de Formação de Facilitador de Educação Permanente em Saúde - unidade aprendizagem - integradora. Brasília (DF): MS; 2006.

17. Ciampone MHT, Melleiro MM. O planejamento e o processo decisório como instrumentos do processo de trabalho gerencial. In: Kurcgant P, coordenador. Gerenciamento em enfermagem. Rio de Janeiro (RJ): Guanabara Koogan; 2005. p.37-53.

18. Cecílio LCO. Uma sistematização e discussão de tecnologia leve de planejamento estratégico aplicado ao setor governamental. In: Merhy EE, Onocko R, organizadores. Agir em saúde: um desafio para o público. São Paulo (SP)/Buenos Aires(AR): Hucitec/ Lugar Editorial; 1997. p.151-67.

19. Gelbecke F, Matos E, Schimidt IS, Mesquita MPL, Padilha MFC. Planejamento estratégico participativo: um espaço para conquista da cidadania profissional. Texto Contexto Enferm. 2006 Jun-Set; 15(3):515-20.

20. Campos RO. Análise do planejamento como dispositivo mediador de mudanças institucionais com base em um estudo de caso. Cad. Saúde Pública 2000 Out-Dez; 16(4):1021-30.

21. Roldão MC. Para um currículo do pensar e do agir: as competências enquanto referencial de ensino e aprendizagem no ensino superior. Workshop competência e avaliação da Universidade de Aveiro; 2005 [acesso 2007 Abr 23]. Disponível em http://www.uc.pt/pessoal/abade/bolonha/docs/ Competencias_roldao.pdf/

22. Hager P, Gonczi, A. What is competence?. Med Teach. 1996 Jan-Mar; 18(1):15-8. 